\title{
IMPACTO AMBIENTAL E RETORNO ACIONÁRIO DE COMPANHIAS LISTADAS NA BM\&FBOVESPA
}

\author{
M. P. LIMA JUNIOR ${ }^{*}$ e V. G. CARVALHO \\ Instituto Federal de Educação, Ciência e Tecnologia do Rio Grande do Norte \\ melquiades.pereira@ifrn.edu.br*
}

Artigo submetido em agosto/2015 e aceito em outubro/2015

DOI: 10.15628/empiricabr.2015.3350

\section{RESUMO}

Este trabalho teve como objetivo analisar se a informação proveniente de um incidente ambiental provoca impacto nos preços de ações das companhias responsáveis, analisando indiretamente a perda de eficiência informacional a nível semi-forte. Para isso, foi utilizada a metodologia de estudos de eventos, juntamente com uma simulação Bootstrap. Os dados de cotações diárias de preços de fechamento dos ativos foram coletados no Software Bloomberg ${ }^{\circledR}$ e as notícias de desastres ambientais foram coletadas em anúncios de jornais. Os eventos se resumiram em companhias do segmento de mineração e petróleo com ações na BM\&FBovespa. Os resultados sugerem evidências de perda de eficiência para os eventos em geral, e que os preços das ações sofreram queda em até 4 dias após o evento. Dessa forma a eficiência do mercado não se comportou de forma semi-forte, pelo atraso máximo de 4 dias na incorporação dessas informações. A pesquisa sugere a necessidade de aumento da credibilidade na divulgação das informações ao mercado, visto que os investidores observam com cautela os efeitos desses eventos nos preços das ações.

PALAVRAS-CHAVE: Eficiência de Mercado, Estudo de evento, Impacto Ambiental, Divulgação das Informações, Mercado de Capitais.

\section{ENVIRONMENTAL IMPACT AND STOCK RETURNS OF COMPANIES LISTED ON BM\&FBOVESPA}

\begin{abstract}
This study aimed to analyze if the information from an environmental incident causes impact on stock prices of the companies responsible, indirectly analyzing the loss of informational efficiency semi-strong level. For this, the event studies methodology was used together with a bootstrap simulation. Data from daily prices of assets were collected from Bloomberg ${ }^{\circledR}$ software and the news of environmental disasters were collected in newspaper advertisements. The events are summarized in the
\end{abstract}

mining and oil sector companies listed on BM\&FBovespa. The results suggest evidence of loss of efficiency for events in general, and that share prices suffered falling within 4 days after the event. Thus market efficiency did not behaved semi-strong form, the delay in the incorporation of this information. Our research suggests a need for increased credibility in the dissemination of information to the market, as investors cautiously observe the effects of these events in stock prices.

KEYWORDS: market efficiency, event study, environmental impact, information flow, capital markets. 


\section{INTRODUÇÃO}

O surgimento da globalização da economia e o desenvolvimento econômico acelerado dos países e das grandes Organizações Multinacionais ocorridos durante o século XX estimularam a preocupação com o meio ambiente. O axioma focado no crescimento econômico passa a se transferir para a expressão do desenvolvimento sustentado.

A ONU e os organismos financiadores internacionais passaram a adotar medidas à submissão de projetos de indústria de base e de infraestrutura à avaliação dos impactos ambientais para financiamentos pelo Banco Interamericano para Reconstrução e Desenvolvimento - BIRD e pelo Banco Interamericano de Desenvolvimento - BID. No Brasil esses estudos foram realizados segundo as normas das agências internacionais, pois há alguns anos atrás o país ainda não dispunha de normas ambientais próprias.

Em 31 de agosto de 1981 foi editada a Lei no 6.938, criando a Política Nacional do Meio Ambiente, estabelecendo conceitos, princípios, objetivos, instrumentos, penalidades, seus fins, mecanismos de formulação e aplicação, instituindo o Sistema Nacional de Meio Ambiente SISNAMA e o Conselho Nacional do Meio Ambiente - CONAMA. Apesar do aumento das normas e da fiscalização ambiental no Brasil, as divulgações referentes às informações de cunho ambiental não são obrigatórias, a Lei 11.638/2007 cujo enfoque se refere à convergência aos International Finance Reporting Standards - IFRS, não contempla os aspectos socioambientais.

Quando se trata de divulgações das informações, mesmo que ambientais, surge a Hipótese de Mercado Eficiente proposta por Fama (1998). A hipótese afirma que o nível de eficiência dos mercados reflete rapidamente as novas informações no preço das ações. A reação aos anúncios das notícias, em mercados eficientes, faz com que os preços das ações se ajustem instantaneamente e reflitam completamente as novas informações. Em outras palavras, não há tendência para aumentos e quedas subsequentes.

Partindo-se desse pressuposto entende-se que as divulgações das informações de cunho ambiental podem conter informações relevantes, que se fornecidas a analistas podem auxiliar na precificação das ações. O objetivo deste trabalho consiste em analisar com base nos preços das ações se o impacto no período do evento ambiental provocou variações no valor da empresa.

\section{REVISÃO BIBLIOGRÁFICA}

Nas últimas décadas houve uma maior ascensão das práticas de evidenciação ambiental, mas existem críticas em relação à forma e o conteúdo das informações ambientais que são divulgados nos relatórios corporativos. As principais falhas detectadas em pesquisas relacionadas à divulgação de informações ambientais são: a falta de uniformidade, profundidade, objetividade, continuidade e credibilidade das informações ambientais divulgadas (BEBBINGTON, GRAY 2001; COSTA, MARION 2007; DEEGAN, GORDON 1996; SKILLIUS, WENNBERG 1998).

Segundo Bebbington e Gray (2001) e Lindstaedt e Ott (2007) a solução para superar as limitações seria regulamentar a divulgação de informações ambientais nos relatórios corporativos. Entretanto as companhias se aparam na teoria dos contratos, e afirmam que a regulamentação aumentaria a competitividade externa e os custos de divulgação. 
Em face desse impasse, de ordem discricionária, as empresas acabam divulgando as informações que acreditam agregar valor, mas quando acreditam que o conteúdo informacional poderá reduzir o valor da ação, as informações por vezes deixam de ser divulgadas. Entretanto, na maioria das vezes o mercado pode absorver a informação mesmo sem ela ser divulgada, e o simples fato da empresa divulgá-la, pode gerar efeitos na percepção do investidor, ou seja, podese sugerir que a divulgação de informações ambientais contribui para aumentar a confiança do acionista nas empresas que divulgam tais informações, elevando o valor da ação.

Em pesquisa realizada por Lindstaedt e Ott (2007), o autor concluiu que apesar dos esforços dos organismos internacionais em determinar normas e estabelecendo diretrizes metodológicas apropriadas à divulgação de informações. A aderência das companhias a tais diretrizes, em média, não atinge $50 \%$ do que é recomendado. No Brasil as divulgações de informações ambientais restringem-se a normas com baixo nível de detalhamento, e o atendimento a tais normas também é parcial.

\section{METODOLOGIA}

\subsection{Estudo de eventos}

Desenvolvido por meio de Fama et al (1969), o estudo de eventos consiste na análise da existência ou não de uma reação significativa nas cotações de ativos de uma ou mais empresas face à ocorrência de um determinado evento, que poderia estar afetando esses valores de mercado. O estudo de eventos é um método, segundo Campbell, Lo e Mackinley (1997) pelo qual é possível medir o efeito de um evento no valor da empresa em estudo. Esse método contribui para análise da racionalidade do mercado, diretamente relacionado ao estudo de Fama (1998).

De acordo com Binder (1998), o estudo de evento é usado em dois tipos de estudos: (1) testar a hipótese nula de que o mercado eficientemente incorpora informações; e, (2) examinar o impacto de um determinado evento na riqueza dos acionistas de uma determinada empresa, mantendo a hipótese de mercado eficiente referente à informação pública.

Recentemente, além da Teoria dos Mercados Eficientes, e da visão comportamental, segundo Lo (2004), há uma nova corrente de pensamento denominada de Teoria dos Mercados Adaptáveis que pode ser vista como uma nova versão da Teoria dos Mercados Eficientes, derivada de princípios evolucionários. Segundo a Teoria proposta por Lo (2004), os preços refletem tanto informações específicas quanto às ditadas pela combinação das condições ambientais. Assim, as preferências agregadas em relação a risco não são constantes universais, mas moldadas pelas forças da seleção natural, o que contribui para o pensamento de que impactos ambientais podem afetar diretamente o valor da empresa. A Teoria dos Mercados Adaptáveis é uma conciliação das duas visões antagônicas anteriores, baseada em uma visão evolutiva das interações econômicas, influenciada por conceitos da economia e da psicologia.

A análise do impacto do evento baseia-se em uma série de retornos antes da ocorrência de determinado evento, então se calcula um retorno esperado para as ações em questão, que deveria ser observado caso o evento não ocorra. Com a definição do que seriam esses retornos normais, compara-se aos retornos efetivamente observados pelo mercado, em busca da identificação ou não de comportamentos anormais de retornos em períodos próximos aos eventos evidenciados. 
As etapas que compõe o estudo de eventos são diagramadas por Campbell, Lo e Mackinley (1997) na Figura 1.

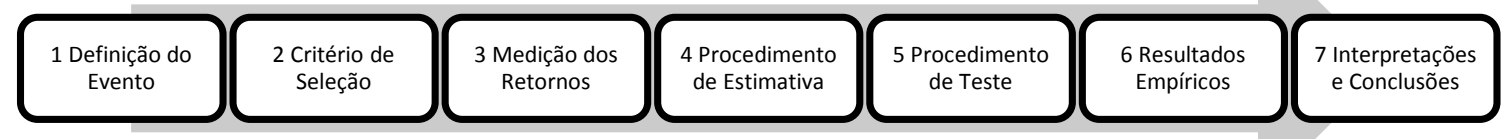

Figura 1 - Etapas do Estudo de Eventos

Fonte: Elaborada com base em Campbell, Lo e Mackinley (1997).

A primeira etapa consiste na definição do evento a ser analisado, em que a sua data é definida como data zero e o período no qual os preços e retornos das ações serão observados é denominado de Janela do Evento. A definição da data zero e da janela do evento é realizada de forma subjetiva pelo pesquisador. Vale ressaltar, que a identificação precisa da data do evento, bem como do período ideal da janela de evento podem contribuir para o resultado da análise, o que demonstra uma limitação do método. Na janela de evento, a quantidade de observações não pode ser curta demais, de modo que não gere perda de informações, insider information, ou longo demais, capturando oscilações não relacionadas ao evento. A Figura 2 demonstra a linha de tempo do estudo do evento.

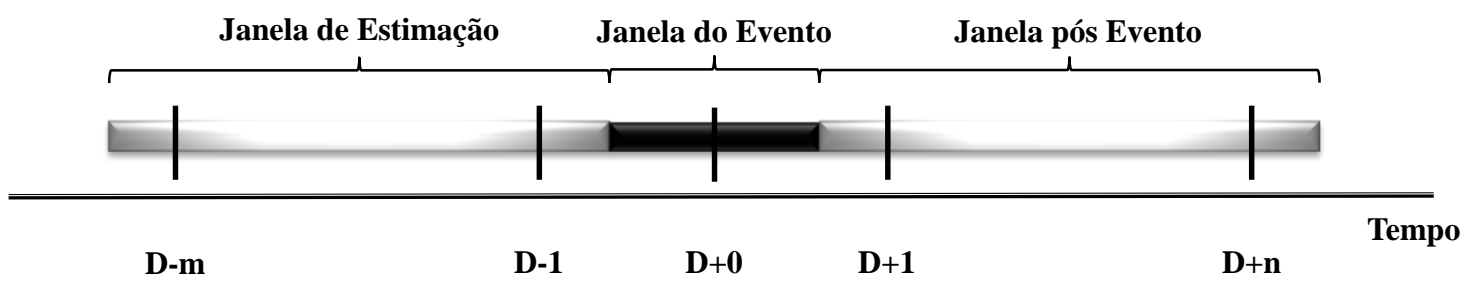

Figura 2 - Linha do tempo para um Estudo de Eventos

Fonte: Elaborada com base em Campbell, Lo e Mackinley (1997).

Conforme a Figura 2, utiliza-se um período prévio, entre $D_{-m}$ e $D_{-1}$, ao evento para realizar a estimação de retornos normais em relação à série em estudo. Esses retornos são comparados com os retornos após o evento, entre o período $D_{+1}$ e $D_{+n}$. Os limites das janelas de tempo, conforme já mencionado, $m$ e $n$, são determinados pelo pesquisador. Para análise e simulação ex post facto há três métodos que serão descritos.

\subsection{Modelos de medição em estudos de eventos}

Buscando observar o impacto do evento nas ações das empresas, faz-se necessário a medição dos retornos anormais $A_{i t}$, conforme Equação 01. Segundo Campbell, Lo e Mackinley (1997) os modelos tratam da diferença entre o retorno ex post facto realmente observado pela ação $R_{i t}$ e seu retorno normal para a mesma data $E\left(R_{i} I X_{t}\right)$. O retorno normal é o retorno esperado para a ação, caso o evento não tivesse ocorrido. $i$ e $t$ identificam respectivamente $o$ ativo e o período de tempo.

$A_{i t}=R_{i t}-E\left(R_{i} I X_{t}\right)$ 
Para cálculo dos retornos anormais, três métodos são abordados por Campbell, Lo e Mackinley (1997). O primeiro, Modelo 1, assume que os retornos normais giram em torno de uma média constante $\overline{\mathrm{R}}_{1}$ dos retornos do próprio ativo, a Equação 02 demonstra o cálculo.

$A_{i t}=R_{i t}-\bar{R}_{l}$

O segundo, Modelo 2, assume que os retornos normais do ativo seguem o comportamento do mercado. Os retornos anormais são calculados por meio da diferença entre os retornos reais e os retornos da carteira de mercado $\mathrm{R}_{\mathrm{mt}}$, na Equação 03 .

$A_{i t}=R_{i t}-R_{m t}$

O terceiro, Modelo 3, relaciona os retornos de uma ação com os retornos da carteira de mercado, também denominado de OLS - Ordinary Last Squares. A relação entre a série do ativo e a série dos retornos da carteira é feita por meio de uma regressão linear entre as variáveis. Segundo Campbell, Lo e Mackinley (1997) a eficiência do modelo depende do $\mathrm{R}^{2}$ da regressão, em outras palavras, depende da aderência do modelo de regressão utilizado. A Equação 04 e 05 demonstram o modelo.

$A_{i t}=R_{i t}-\hat{\alpha}_{i}-\hat{\beta}_{i} R_{m t}$

$R_{i t}=\hat{\alpha}_{i}-\hat{\beta}_{i} R_{m t}+\varepsilon_{i t}$

As variáveis $\widehat{\alpha}_{i}$ e $\widehat{\beta}_{\mathrm{i}}$ são os parâmetros de uma regressão linear simples, respectivamente coeficiente linear e angular da reta incluindo um erro $\varepsilon_{\mathrm{it}}$, Considerando ainda que $\mathrm{E}\left[\varepsilon_{\mathrm{it}}\right]=0$ e $\operatorname{Var}\left[\varepsilon_{\mathrm{it}}\right]=\sigma_{\varepsilon i t}^{2}$. As variáveis são calculadas para o período da janela de estimação selecionado. Esse último modelo pressupõe os mesmos requisitos de uma regressão: normalidade, linearidade, estacionariedade, independência dos resíduos e estabilidade da variância dos resíduos.

Após a seleção do modelo e dos retornos normais segue a etapa de estimação dos parâmetros, realizada por meio da seleção da janela de estimação. Conforme Campbell, Lo e Mackinley (1997) não devem ser incluídas no procedimento de estimação os valores da data 0 nem os valores, referente a janela do evento, nem os valores da janela pós evento. Por último, no procedimento de teste, é realizada uma análise, por meio de um teste estatístico, para confirmação de que os retornos anormais para a janela de evento possuem média aproximadamente zero, o que confirma que o evento não absorveu impacto no evento selecionado.

\section{RESULTADOS E DISCUSSÕES}

Para alcançar o objetivo do trabalho, foi realizada uma pesquisa empírica no formato ex post facto, que consiste em uma simulação dos modelos no passado. A pesquisa foi quantitativa por se tratar da observação de resultados numéricos do estudo conforme descrito em Mahoney e Goertz (2006), o que influenciou na forma de avaliação. A avaliação desses resultados foi realizada por meio da análise da sensibilidade dos modelos, utilizando gráficos demonstrativos.

Os dados coletados foram obtidos de origem secundária por meio do software Economática $^{\circledR}$. A seleção da amostra para subsidiar a elaboração do modelo compreendeu o 
histórico de fechamentos diários ajustados por proventos, bonificações, splits e inplits. Os ativos selecionados foram das empresas relacionadas aos eventos utilizados para o estudo no respectivo ano de ocorrência. Também foram utilizados anúncios de jornais, referente a notícias de desastres ambientais provocados por companhias com ações na Bovespa, coletados no Bloomberg ${ }^{\circledR}$. Inicialmente foi realizada uma análise qualitativa dos dados encontrados para em seguida fazer uma análise quantitativa por meio de estudo de eventos. A tabulação e o tratamento dos dados foram realizados de forma estatística com uso do Software $R$.

Os eventos foram pesquisados de acordo com o impacto ambiental, o segmento de mineração e petróleo e a disponibilidade de informações sobre o evento, bem como a presença da companhia na bolsa de valores. Os eventos selecionados foram organizados na Tabela 1. A data do evento foi utilizada como data 0 na análise e as janelas de tempo utilizadas foram os preços pertencentes ao período do ano do evento em questão.

Na Tabela 1, a data corresponde a $\mathrm{D}_{0}$ na análise do evento. O primeiro evento na área de Petróleo tem como responsável apenas uma empresa da área, e o segundo corresponde a quatro empresas da área de mineração. O valor da multa corresponde ao valor do documento do auto de infração e por último o motivo do impacto. Para análise, foram selecionados dois ativos do primeiro evento PETR3 e PETR4, e do segundo evento foram selecionados três ativos VALE3, VALE5 e CSNA3 respectivamente na ordem da Tabela 1. Os preços diários coletados do primeiro evento foi entre 03 de janeiro a 29 de dezembro do ano de 2000 e o segundo evento foram coletados entre 03 de janeiro a 31 de outubro de 2011.

Tabela 1 - Descrição dos Eventos Selecionados

\begin{tabular}{c|c|c|c|c|c}
\hline Evento & Data & Empresas & Segmento & Multa & Motivo \\
\hline 1 & $16 / 06 / 00$ & Petrobras & Petróleo & $\mathrm{R} \$ 50.000 .000,00$ & $\begin{array}{c}\text { Derramamento de óleo no } \\
\text { Rio Iguaçu, Araucária-PR }\end{array}$ \\
\hline 2 & $02 / 08 / 11$ & Vale & Mineradora & $\mathrm{R} \$ 1.300 .000,00$ & $\begin{array}{c}\text { Pó que encobriu a Cidade } \\
\text { de Congonhas -MG }\end{array}$ \\
\hline 2 & $02 / 08 / 11$ & $\mathrm{CSN}$ & Mineradora & $\mathrm{R} \$ 1.300 .000,00$ & $\begin{array}{c}\text { Pó que encobriu a Cidade } \\
\text { de Congonhas }-M G\end{array}$ \\
\hline
\end{tabular}

Para início da análise, os preços foram organizados em gráficos de linha e confrontados com o Índice Bovespa que é o indicador macro do mercado brasileiro. A Figura 3 representa os preços da área de petróleo, referente ao evento 1 da Tabela 1, os ativos da empresa responsável foram PETR3 e PETR4 no ano de 2000. O segundo evento, demonstrado pela Figura 4, representa os preços dos ativos VALE3, VALE5 e CSNA3, também confrontados com o comportamento do mercado, mais recentemente no ano de 2011. 


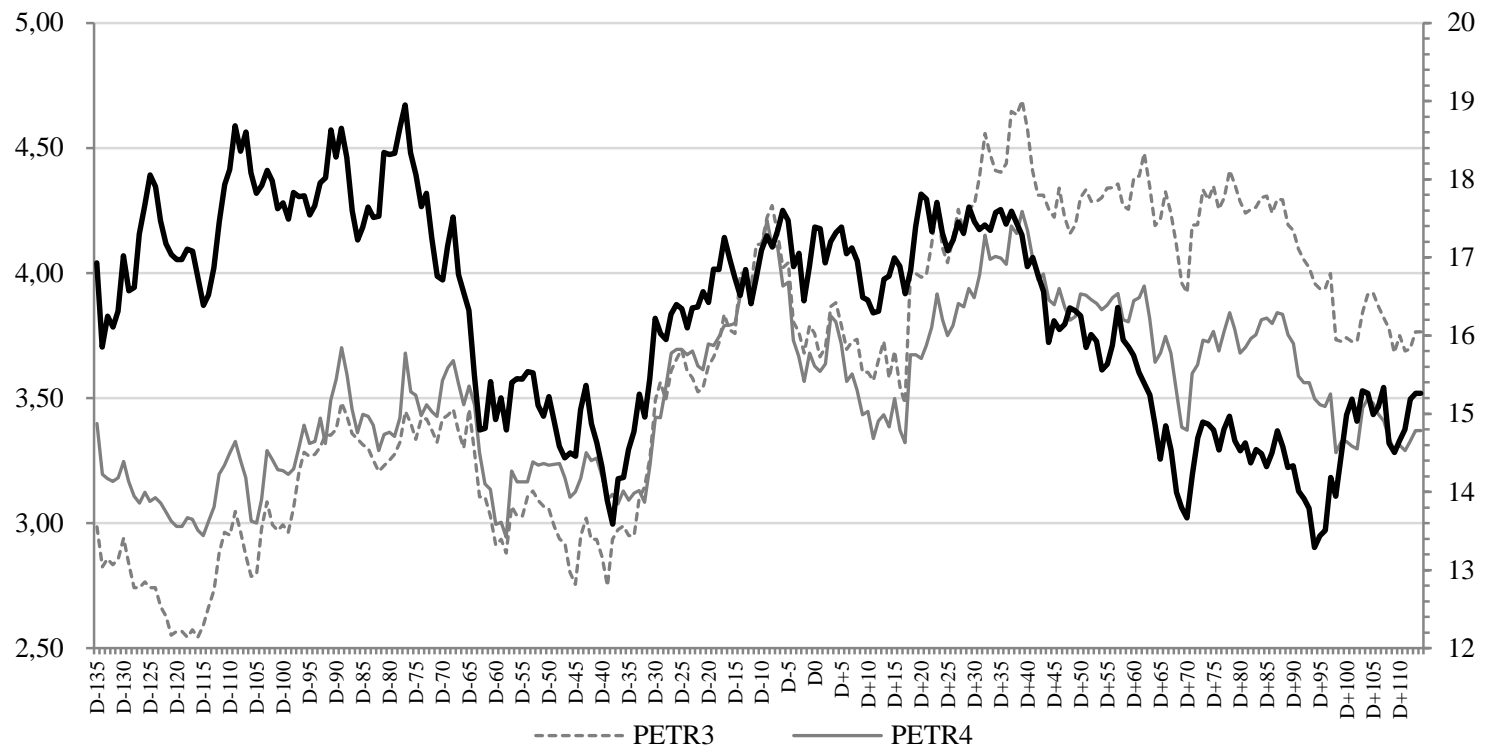

Figura 3 - Preços dos ativos do evento na área de Petróleo e o Índice Bovespa

A primeira análise, dos preços demonstrou que o primeiro evento, não demonstrou comportamento atípico capaz de demonstrar que houve ou não algum indício do impacto do evento. No segundo, no período de $D_{0}$ percebe-se visualmente que houve uma queda repentina dos preços no período, porém além dos ativos, o índice de mercado também obteve queda de pontuação de $8,08 \%$. Em busca de informações no período, constatou-se que a queda em 08 de agosto de 2011 em $D_{+4}$ não foi referente ao evento ambiental, mas sim de um impacto em várias bolsas decorrentes do rebaixamento da nota de risco dos EUA pela Standard \& Poor's - S\&P de AAA para $A A+$, o que influenciou no comportamento dos ativos selecionados. No período outros índices mundiais obtiveram quedas significativas, como Dow Jones, Nasdaq, S\&P, FTSEurofirst, $\mathrm{MSCl}$ e Nikkei.

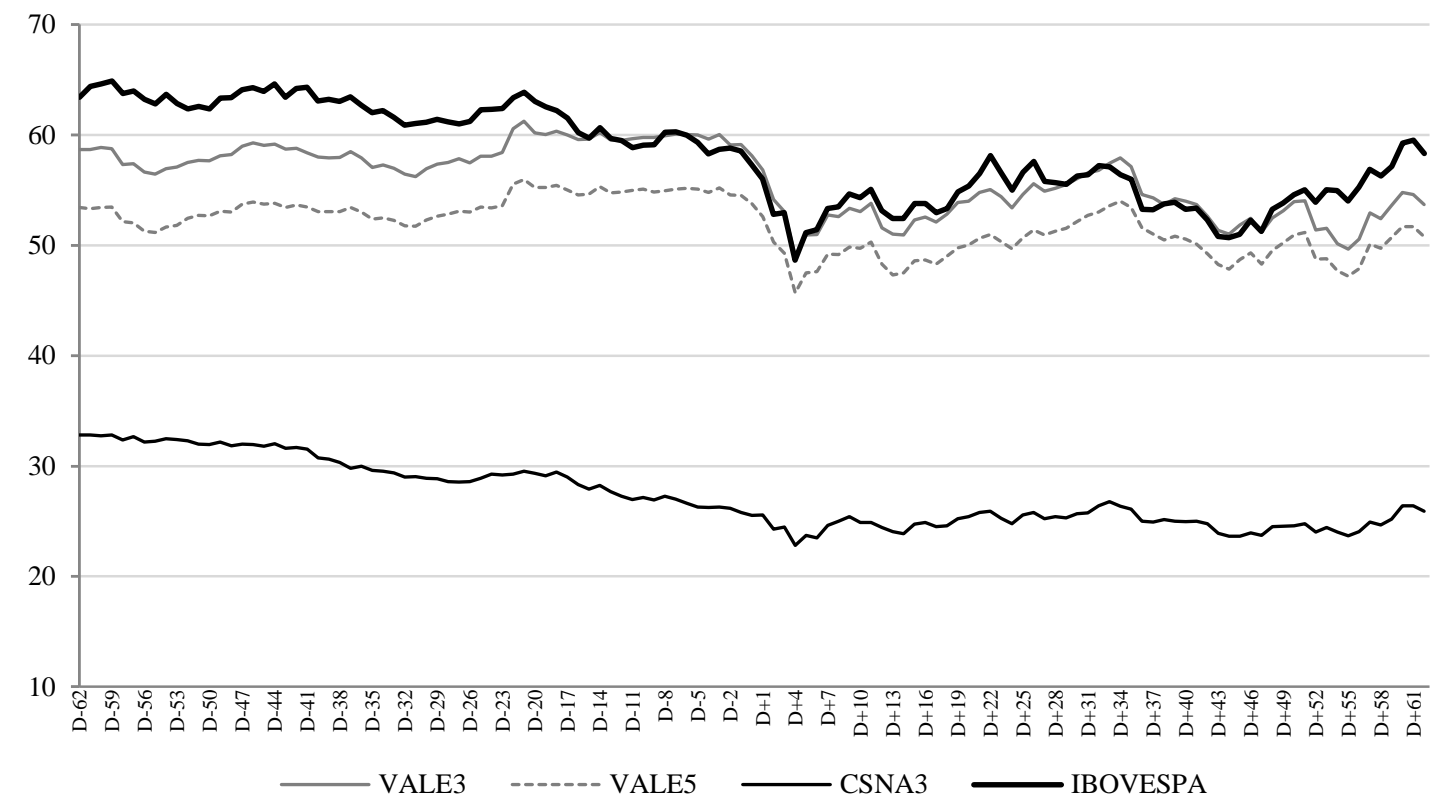

Figura 4 - Preços dos ativos do evento na área de Mineração e o Índice Bovespa 
Os resultados da análise gráfica dos preços demonstraram a necessidade da utilização de modelos mais sofisticados como os de estudos de eventos, baseados nos retornos dos ativos. Para análise, conforme Campbell, Lo e Mackinley (1997), foram utilizados como referência três modelos de avaliação. A validação dos modelos baseia-se na expectativa de que a média dos retornos anormais calculados $A_{i t}$ é diferente de zero, a qual demonstra que os retornos foram influenciados e que não seguiram seu comportamento normal. As conclusões de cada modelo foram analisadas separadamente.

A partir dos preços ajustados dos ativos, foram calculados os retornos contínuos para aplicação dos modelos de estudo de eventos. No Modelo 1, além da aplicação da solução da Equação 02, os retornos anormais $A_{\text {it }}$ foram calculados com base em quatro períodos de janela de estimação, para 30, 60, 90 e 120 no período anterior a $D_{0}$, pois conforme Binder (1998) o período de estimação influencia nos resultados. Para o horizonte de da janela pós evento, foi utilizado o restante dos dados até o final do ano para análise. Para os testes foi elaborada a Tabela 2 demonstra os valores $p$ da estatística do teste $t$ de cada ativo em cada evento estudado. A hipótese $\mathrm{H}_{1}$ analisou se a média dos retornos anormais é aproximadamente zero, o que demonstra a não interferência na série.

Tabela 2 - Valor $\mathbf{p}$ da estatística do teste $\mathbf{t}$ do Modelo 1

\begin{tabular}{c|c|c|c|c|c}
\hline p-valor & \multicolumn{2}{|c|}{ Evento 1 } & \multicolumn{3}{c}{ Evento 2 } \\
\hline Janela Estimação & PETR3 & PETR4 & VALE3 & VALE5 & CSNA3 \\
\hline 30 & $0,02^{* * *}$ & $0,01^{* * *}$ & 0,62 & 0,40 & 0,18 \\
\hline 60 & 0,18 & 0,16 & 0,78 & 0,60 & 0,20 \\
\hline 90 & 0,48 & 0,42 & 0,89 & 0,75 & 0,31 \\
\hline 120 & 0,19 & 0,22 & 0,94 & 0,91 & 0,35 \\
\hline
\end{tabular}

Os resultados do primeiro modelo demonstraram que apenas o primeiro evento, usando uma janela de estimação para trinta dias demonstrou impacto nos preços das ações. Consequentemente, os retornos das ações da área de petróleo, com parâmetros calculados em trinta dias obtiveram impacto, com uma média dos retornos anormais diferentemente de zero a $5 \%$ de significância.

Como os retornos anormais do Modelo 2 não considera as observações passadas, não foi considerada janelas de tempo, pois trata apenas da diferença entre os retornos dos ativos e os retornos do mercado. Os resultados do segundo método demonstraram que não houve diferença significativa entre os retornos anormais dos ativos, ou seja, a média é igual à zero. $O$ resultado mostra que as séries não obtiveram influência significativa tomando como base o Índice Bovespa como mercado. A Tabela 3 representa os resultados do segundo modelo aplicado.

Tabela 3 - Valor p da estatística do teste t do Modelo 2

\begin{tabular}{c|c|c|c|c|c}
\hline p-valor & \multicolumn{2}{|c|}{ Evento 1 } & \multicolumn{3}{c}{ Evento 2 } \\
\hline Janela Estimação & PETR3 & PETR4 & VALE3 & VALE5 & CSNA3 \\
\hline- & 0,76 & 0,90 & 0,83 & 0,92 & 0,25 \\
\hline
\end{tabular}

$\mathrm{Na}$ análise do terceiro modelo, foi possível executar a regressão dos dados para os quatro períodos de janelas de estimação. Conforme sugerido por Campbell, Lo e Mackinley (1997), para validação do modelo de regressão a Tabela 4 apresenta os coeficientes de determinação dos modelos de regressão executados. Na comparação entre os eventos, o segundo apresentou 
coeficientes de determinação mais altos, indicando que os modelos de regressão foram mais precisos na explicação dos ativos.

Tabela 4 - Valor p da estatística do teste t do Modelo 2

\begin{tabular}{c|c|c|c|c|c}
\hline $\mathrm{R}^{2}$ & \multicolumn{2}{|c|}{ Evento 1 } & \multicolumn{3}{c}{ Evento 2 } \\
\hline Janela Estimação & PETR3 & PETR4 & VALE3 & VALE5 & CSNA3 \\
\hline 30 & 0,261 & 0,340 & 0,405 & 0,426 & 0,546 \\
\hline 60 & 0,329 & 0,331 & 0,442 & 0,451 & 0,469 \\
\hline 90 & 0,307 & 0,361 & 0,422 & 0,423 & 0,387 \\
\hline 120 & 0,296 & 0,365 & 0,482 & 0,472 & 0,441 \\
\hline
\end{tabular}

Após a modelagem econométrica, foram elaborados os retornos anormais dos ativos de ambos os eventos. Os resultados, demonstrados na Tabela 5, apresentaram evidências do impacto da divulgação apenas para o segundo evento, usando uma janela de estimação de até 30 dias, o que remete a perda de eficiência informacional. No uso de janelas de estimação mais longas, todos os modelos apresentaram sensibilidade ao período selecionado, em períodos maiores os modelos perderam qualidade na elaboração dos retornos anormais, corroborando com Binder (1998).

Tabela 5 - Valor p da estatística do teste t do Modelo 2

\begin{tabular}{c|c|c|c|c|c}
\hline p-valor & \multicolumn{2}{|c|}{ Evento 1 } & \multicolumn{3}{c}{ Evento 2 } \\
\hline Janela Estimação & PETR3 & PETR4 & VALE3 & VALE5 & CSNA3 \\
\hline 30 & 0,385 & 0,293 & $0,006^{* * *}$ & $0,014^{* *}$ & $0,017^{* *}$ \\
\hline 60 & 0,433 & 0,425 & $0,081^{*}$ & 0,136 & $0,057^{*}$ \\
\hline 90 & 0,553 & 0,747 & 0,140 & 0,192 & 0,189 \\
\hline 120 & 0,202 & 0,410 & 0,247 & 0,331 & 0,120 \\
\hline
\end{tabular}

Em geral, os modelos com diferentes metodologias apresentaram diferentes resultados na conclusão do estudo de eventos para impactos ambientais. O primeiro modelo de média apresentou evidências de perda de eficiência semi-forte para o primeiro evento na área de petróleo a 5\% de significância, o segundo modelo de mercado, não apresentou perda de eficiência informacional e por último, o terceiro modelo de regressão apresentou perda de eficiência informacional para o segundo evento a $5 \%$ de significância.

Considerando que os modelos apresentaram significância para 30 dias de janela, realizouse uma análise robusta para validar o efeito significativo da distorção entre esses dias. Os retornos acumulados foram calculados para todas as séries, a função faz simulações com o método Bootstrap para gerar distribuição de média de todo o retorno acumulado das séries temporais. Foi utilizado o pacote "eventstudies" do software R, com a função "inference.Ecar" (DAVINSON; HINKLEY; SCHECHTMAN, 1986).

A Figura 5 consiste em um conjunto de gráficos que resumem o comportamento dos retornos acumulados da simulação. O intervalor de confiança foi entre 2,5 e 97,5. O gráfico A consiste na simulação entre -5 e +5 dias, o gráfico $B$ entre -10 e +10 dias, o gráfico $C$ entre -20 e +20 dias e o gráfico $D$ entre -30 e +30 dias. 
A

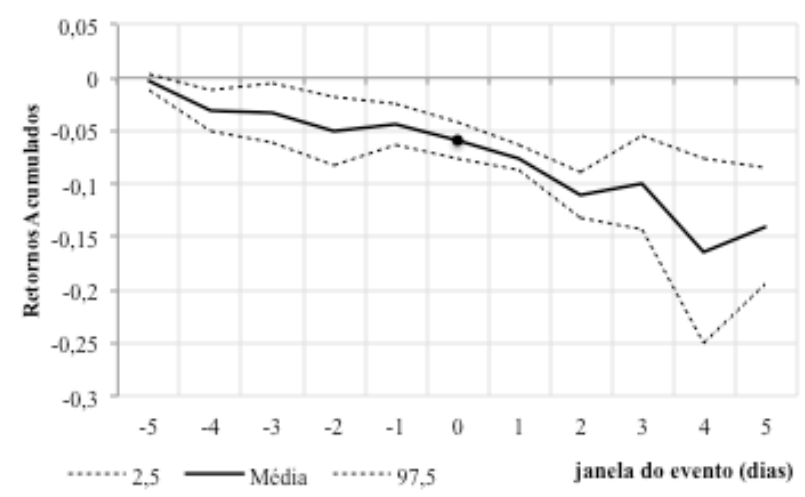

C

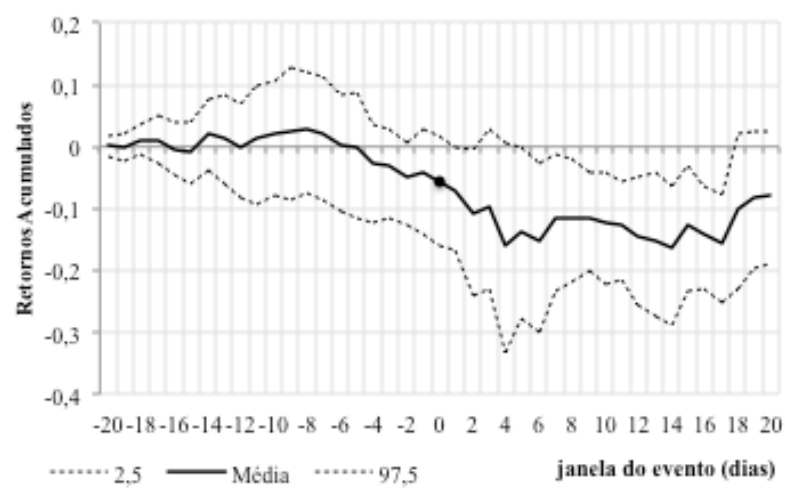

B

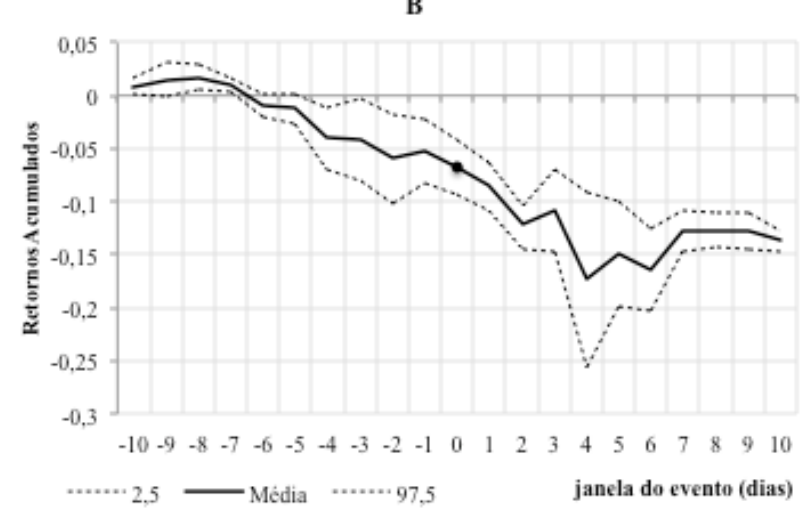

D

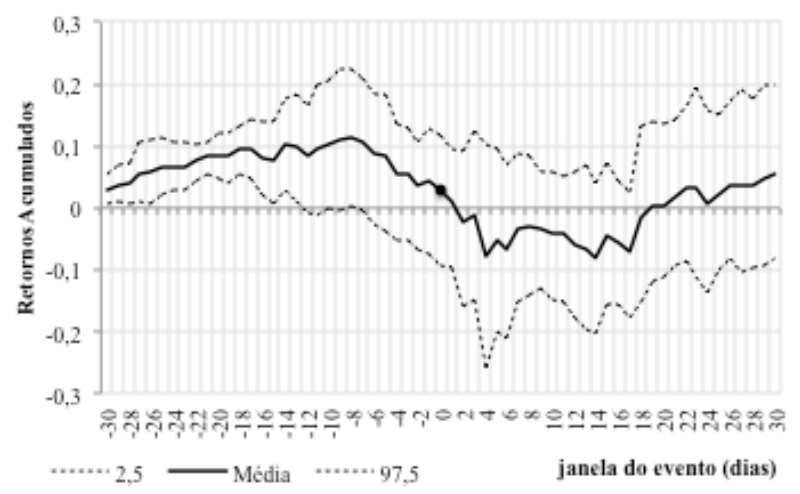

Figura 5 - Média dos retornos acumulados com o intervalo de confiança entre 2,5 e 97,5.

Nos gráficos A e o B da figura 5, percebe-se um efeito conjunto em torno do $4^{\circ}$ dia após o evento no dia 0.0 que demonstra que o mercado sofreu os efeitos dos impactos ambientais em 4 dias, após os efeitos das divulgações na mídia. Os gráficos $C$ e $D$ da figura 5 , demonstram que após - $4^{\circ}$ dia os retornos tendem a retornar ao equilíbrio, o que colabora com o argumento de Fama (1969) de que os efeitos nos retornos se dissipam até o $5^{\circ}$ dia posterior ao evento.

\section{CONCLUSÃO}

Esse trabalho teve como objetivo analisar com base nos preços das ações se o impacto no período do evento ambiental provoca variações no valor da empresa. Esse estudo analisa indiretamente a perda de eficiência informacional a nível semi-forte conforme estudo de Fama (1969). Em outras palavras, observa-se se uma informação proveniente de um incidente ambiental provoca impacto nos preços de ações das companhias responsáveis em até 4 dias após o evento.

Os resultados apresentaram evidências de perda de eficiência para o primeiro evento, no modelo de média e para o segundo evento, no modelo de regressão, ambos dentro dos trinta dias de período de estimação. Essas evidências empíricas mostram que, apesar dos avanços, o mercado de capitais brasileiro não se comportou de maneira eficiente na divulgação de eventos ambientais no período analisado, no que se refere à forma semi-forte. A validação robusta confirmou esses efeitos em até 4 dias após o evento.

Como tais estudos ainda não observaram esses tipos de eventos, comparamos com os resultados de outros tipos de eventos como fusões e aquisições, anúncios de boas práticas de 
governança corporativa, anúncios de mudança cambial e decisões de investimentos. Os resultados de perda de eficiência informacional e ajustes lentos e graduais aos preços corroboram com os resultados encontrados em Camargos e Barbosa (2006), Perobelli et al (2000), Lucchesi e Famá (2007) e Terra e Lima (2006) e contraria os resultados de mercado eficiente de Batistella et al (2004).

Por fim, o trabalho também demonstrou que o mercado no curto prazo sofreu perturbações provenientes de fatores ambientais. Em complemento ao estudo de Lindstaedt e Ott (2007), as empresas brasileiras deveriam observar com mais cautela fatores ambientais, principalmente por afetar o valor da empresa no mercado. $O$ trabalho também complementa a crítica realizada por Costa e Marion (2007), que observam a necessidade de maior credibilidade às informações, visto que os investidores observam com cautela os efeitos desses eventos nos preços das ações.

Para futuros trabalhos, algumas sugestões foram pontuadas como um estudo mais aprofundado acerca dos motivos pelos quais os anúncios dos impactos ambientais ao longo do tempo refletiram de maneira contundente nos preços das ações, incluindo a forma com que esses anúncios são realizados, por meio de terceiros ou pela própria companhia.

\section{REFERÊNCIAS}

1. BATISTELLA, F. D. et al. Retornos de ações e governança corporativa: um estudo de eventos. In: Congresso de Controladoria e Contabilidade, 4, 2004, São Paulo. Anais... São Paulo: FEA/USP, 2004.

2. BEBBINGTON, J.; GRAY, R. An Account of Sustainability: Failure, Success and a Reconceptualization. Critical Perspectives on Accounting, v. 12, n. 5, p. 557-588, 2001.

3. BINDER, J. The Event Study Methodology Since 1969. Review of Quantitative Finance and Accounting, v. 11, n. 2, p. 111-137, 1998.

4. CAMARGOS, M. A. D.; BARBOSA, F. V. Eficiência informacional do mercado de capitais brasileiro pós-Plano Real: um estudo de eventos dos anúncios de fusões e aquisições. Revista de Administração, v. 41, p. 43-58, 2006.

5. CAMPBELL, J.Y.; LO, A.W.; MACKINLAY, A.C. The Econometrics of Financial Markets. Princeton: Princeton University Press, 1997.

6. COSTA, R. S. D.; MARION, J. C. A uniformidade na evidenciação das informações ambientais. Revista Contabilidade \& Finanças, v. 18, p. 20-33, 2007.

7. DAVINSON, A.; HINKLEY, D.V.; SCHECHTMAN, E. Efficient bootstrap simulation. Biometrika, v.73, n.3, p. 555-566, 1986.

8. DEEGAN, C.; GORDON, B. A study of the environmental disclosure practices of Australian corporations. Accounting and Business Research, v. 26, p. 187-199, 1996.

9. FAMA, E.F. et al. The Adjustment of Stock Prices to New Information. International Economic Review, Vol. 10, Feb, 1969.

10. FAMA, E.F. Market efficiency, long-term returns, and behavioral finance. Journal of Financial Economics, v. 49, n. 3, p. 283-306, 1998. 
11. LINDSTAED, A.R.S.; OTT, E. Evidenciação de Informações Ambientais pela Contabilidade: um Estudo Comparativo entre as Normas Internacionais (ISAR/UNCTAD), Norte Americanas e Brasileiras. Contabilidade Vista e Revista, v.18, n.4, p. 11-35, 2007.

12. LO, A.W.C. Adaptive Markets Hypothesis: Market Efficiency from an Evolutionary Perspective. Journal of Portfolio Management, Vol. 30, n.5, p. 15-29, 2004.

13. LUCCHESI, E. P.; FAMÁ, R. O impacto das decisões de investimento das empresas no valor de mercado das ações negociadas na Bovespa no período de 1996 a 2003. Revista de Administração, v. 42, p. 249-260, 2007.

14. MAHONEY, J.; GOERTZ, G. A Tale of Two Cultures: Contrasting Quantitative and Qualitative Research. Political Analysis, v. 14, n. 3, p. 227-249, Summer, 2006.

15. PEROBELLI, F. F. C. et al. Expectativas racionais e eficiência informacional: análise do mercado acionário brasileiro no período 1997-1999. Revista de Administração Contemporânea, v. 4, p. 7-27, 2000.

16. SKILLIUS, A; WENNBERG, U. Continuity, Credibility and Comparability: Key challenges for corporate environmental performance measurement and communication. The International Institute for Industrial Environmental Economics at Lund University. Feb. 1998.

17. TERRA, P. R. S.; LIMA, J. B. N. D. Governança corporativa e a reação do mercado de capitais à divulgação das informações contábeis. Revista Contabilidade \& Finanças, v. 17, p. 35-49, 2006. 